\title{
A EVOLUÇÃO DO BALANCED SCORECARD - UMA COMPARAÇÃO COM OUTROS SISTEMAS
}

\author{
H. I. Saraiva ${ }^{*}$, MC. F. G. Alves •• \\ "Instituto Politécnico da Guarda, "Universidade da Beira Interior \\ helenasaraiva@ipg.pt
}

Submetido 06/12/ 2016 - Aceito 08/07/2017

DOI: $10.15628 /$ holos.2017.5438

\section{RESUMO}

No presente trabalho efetuou-se uma análise da evolução histórica do Balanced Scorecard (BSC) comparando a evolução desta ferramenta de gestão com a evolução de outras ferramentas suas contemporâneas. Nesta análise foi demonstrado que a evolução sofrida pelo BSC estará muito relacionada com o facto de o conceito sempre ter tido, ao longo do tempo e desde a sua aparição, uma ampla aplicação prática. Visou também analisar-se o efeito que a utilização deste instrumento introduziu nas práticas de gestão das organizações.

O trabalho foi desenvolvido através de análise bibliográfica dos principais textos publicados pelos autores do Balanced Scorecard e por outros que aportaram contribuições importantes para o tema em análise, assim como da constatação relativa ao facto de os autores do conceito sempre terem desenvolvido a aplicação empírica da sua metodologia, contribuindo para a sua disseminação e esta para a sua evolução.

Assim considera-se poder concluir que a utilização prática do conceito influenciou a evolução do Balanced Scorecard e, vice-versa, a evolução do mesmo também fez alterar o seu campo de aplicação, a avaliar pelas evoluções mais recentes.

PALAVRAS-CHAVE: Balanced Scorecard, evolução, aplicação prática, IAM- Intangible Assets Monitor, Skandia Navigator.

\section{THE EVOLUTION OF THE BALANCED SCORECARD - A COMPARISON WITH OTHER SYSTEMS}

\section{ABSTRACT}

This paper makes an analysis of the historical evolution of the Balanced Scorecard (BSC), comparing the evolution of this management tool with the evolution of his other contemporary tools. This analysis shows that the evolution experienced by the BSC will be closely related to the fact that the concept has always had, over time, and since its appearance, a wide practical application. We also aimed to analyze the effect that the use of this management tool introduced in the organizations management practices.

The work was developed through an extensive analysis of the main texts published by the authors of the
Balanced Scorecard and others who made important contributions to the subject in question as well as the finding on the fact that the concept always had developed with the contributions from empirical application, contributing to its spread and to this evolution.

So we can conclude that the practical use of the concept has influenced the evolution of the Balanced Scorecard, this because it is clear that over time the use given to the BSC has evolved, and vice versa, the evolution of the tool did also changed its own scope, judging by recent developments.

KEYWORDS: Balanced Scorecard, evolution, practical use, IAM-Intangible Assets Monitor, Skandia Navigator. 


\section{Introdução}

A partir do final do século XX, nomeadamente a partir da década de oitenta, foi identificada a necessidade de alterar a forma de avaliar o desempenho das organizações, mais concretamente no que se referia à utilização praticamente exclusiva de indicadores financeiros. Neste contexto surge o modelo de Balanced Scorecard (BSC), por Kaplan e Norton (1992).

Este modelo e o respetivo conceito foi evoluindo ao longo do tempo, em consequência do tipo de utilização efetuada na prática pelas organizações, de acordo com as fases listadas por Saraiva (2011a, 2011b) e por Saraiva e Alves (2015):

- sistema de medição de desempenho: quando consiste num conjunto de objectivos e indicadores com uma orientação específica - correspondente ao período inicial de aparecimento do BSC - entre 1992 e 1993;

- sistema de comunicação: sempre que é utilizado para divulgar a todos os elementos da organização quais os objectivos estratégicos da mesma - entre 1993 e 1996/97;

- sistema de avaliação global e individual: o BSC começa a delinear-se como ferramenta de implementação de estratégias, transformando-as em medidas de acções específicas e criando valor nos intangíveis - entre $96 / 97$ e o ano de 2004;

- sistema de gestão estratégica: utilizado como elemento de suporte à tomada de decisão e de apoio à gestão da organização - isto no período que medeia entre 2004 e 2008;

- sistema de gestão estratégico que gere/avalia o próprio sistema de Gestão - integrando as componentes do capital intelectual das organizações na prossecução da estratégia e iniciando um caminho de aparente "viragem para o exterior" - a partir de 2008;

- sistema de gestão de alianças estratégicas, assumindo um papel nas relações com o exterior da organização, na gestão de alianças estratégicas - a partir de 2010.

Simultaneamente à aparição do conceito de BSC, surgiram outros conceitos semelhantes, entre os quais se destacaram na literatura os correspondentes às metodologias Intangible Assets Monitor (IAM), (Sveiby, 1987) e Skandia Navigator, (Edvinsson, 1997).

Sendo de evidenciar que entre os modelos aqui comparados um deles é anterior à aparição do BSC e outro posterior, todos abordavam uma realidade que era premente à época: a questão da valorização e quantificação de aspetos não financeiros inerentes ao funcionamento das organizações e fundamentais à explicação da formação dos resultados dessas mesmas organizações. Em termos cronológicos, o BSC surge exatamente no ponto médio, relativamente à data da aparição dos outros dois modelos aqui analisados.

Apesar desta coincidência em termos dos momentos de sua criação, cerca de vinte e cinco anos após a aparição do BSC, verifica-se que a sua aplicação se disseminou em termos globais, enquanto os outros dois modelos analisados foram sendo, progressivamente, relegados a um segundo plano. Cremos que isto se deve essencialmente ao facto de o BSC ter tido desde a sua génese uma ampla aplicação e de essa aplicação se ter tornado crescente ao longo do tempo, ao invés do que aconteceu com os outros dois conceitos.

Assim, para fundamentar o tipo de evolução identificado, o presente trabalho foi organizado de acordo com a seguinte subdivisão: breve caraterização dos três modelos, análise da sua evolução e correspondentes conclusões. A ordem de apresentação dos modelos será 
precisamente a ordem cronológica, uma vez que os objetivos deste trabalho estão essencialmente relacionados com a evolução cronológica do BSC.

A análise efetuada será essencialmente baseada em pesquisa bibliográfica e na comparação analítica entre conceitos e respetivas evoluções identificadas.

\section{1 - O Modelo Intangible Assets Monitor (IAM)}

O modelo IAM, anterior no tempo ao conceito de BSC, focaliza-se na avaliação dos ativos intangíveis, assim como na comunicação da estratégia, a qual é também assumida (tal como no $\mathrm{BSC})$, como um elemento central.

O IAM tem como finalidade principal a avaliação e evolução dos activos intangíveis, atribuíndo ao fator capital humano o aspecto essencial da competitividade organizacional.

Por regra, consiste num dispositivo onde são apresentados os três grupos que formam o conjunto dos ativos intangíveis, classificados por perspectivas ou categorias cuja finalidade é a de caraterizar esses ativos. O dispositivo deve ser acompanhado por comentários, efetuando-se uma selecção de indicadores entre os principais identificados para cada categoria.

Assim, um número reduzido de indicadores é identificado ou definido, concretamente um ou dois de entre os indicadores de referência, sendo associados a quatro diferentes categorias ou perspectivas estruturantes do modelo:

- crescimento,

- mudança/inovação,

- eficiência e

- estabilidade/risco.

Na figura seguinte (figura 1), é ilustrado graficamente o modelo IAM.

Figura 1. Representação gráfica do modelo Intangible Assets Monitor.

Intangible Assets Monitor

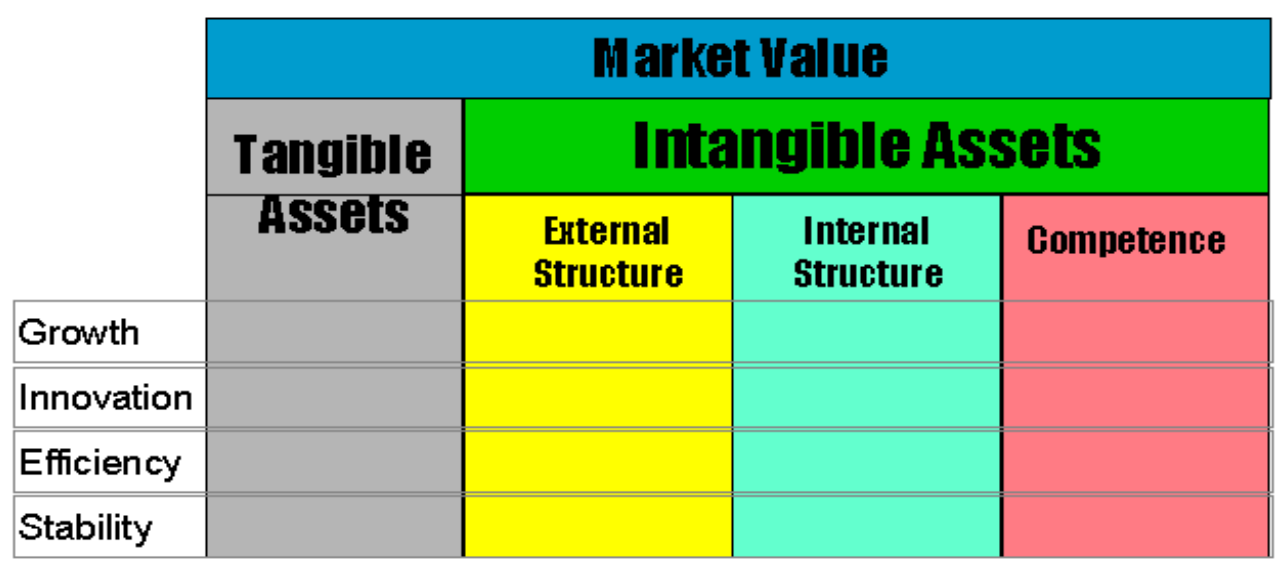

○ Karl Erik Sveiby 1999-2001 all rights reserved

Fonte: http://www.sveiby.com/articles/MeasurelntangibleAssets.html, consultado em Julho de 2011

O modelo distingue claramente entre ativos tangíveis e intangíveis, classificando os intangíveis como ativos reais e proporcionando a perceção sobre temas como a mudança e a 
evolução dos fluxos de conhecimento, consubstanciados nas perspectivas de crescimento, inovação/renovação, eficiência ou utilização e risco versus estabilidade, aplicadas à evolução do ativo intangível considerado.

Os ativos intangíveis encontram-se, segundo esta metodologia, divididos em três grupos (Viedma Martí, 2002):

- a estrutura externa do IAM compreende marcas, relações com clientes, fornecedores e outros stakeholders externos; este grupo é também designado por Capital Relacional;

- a estrutura interna é composta por práticas de gestão da organização, estrutura legal, investigação e desenvolvimento, software, bases de dados e cultura da empresa; este grupo é habitualmente conhecido por Capital Estrutural;

- as competências dos recursos humanos que integram know-how, conhecimentos, educação e experiência das pessoas que trabalham na organização e que correspondem ao grupo normalmente referenciado como Capital Humano.

Os indicadores de referência são apresentados na Figura 2, onde constam os associados a cada grupo e perspectiva do IAM.

Figura 2 - Modelo Intangible Assets Monitor e Indicadores.

\begin{tabular}{|c|c|c|}
\hline $\begin{array}{c}1.1 .1 \quad \begin{array}{l}\text { Indicadores de Estrutura } \\
\text { Externa }\end{array} \\
\text { (marcas, relações com clientes e fornecedores) }\end{array}$ & $\begin{array}{r}1.1 .2 \quad \begin{array}{c}\text { Indicadores de } \\
\text { Estrutura Interna }\end{array} \\
\text { (Gestão, estrutura legal, I\&D, Software) }\end{array}$ & $\begin{array}{c}\text { 1.1.3 Indicadores de Competências dos } \\
\text { Recursos Humanos }\end{array}$ \\
\hline $\begin{array}{c}\text { 1.1.3.1 Indicadores de Crescimento } \\
\text { Crescimento Orgânico } \\
\text { Rentabilidade por cliente }\end{array}$ & $\begin{array}{l}\text { 1.1.3.2 Indicadores de Crescimento } \\
\text { Investimento em TI } \\
\text { Investimentos em Estrutura Interna }\end{array}$ & $\begin{array}{l}\text { 1.1.3.3 } \begin{array}{c}\text { Indicadores de Crescimento } \\
\text { Indíce de Competência }\end{array} \\
\frac{\mathrm{N}^{\mathrm{o} \text { de anos na profissão }}}{\text { Nível de Instrução. }} \\
\text { Turnover de Competências. }\end{array}$ \\
\hline $\begin{array}{l}\text { 1.1.3.4 Indicadores de Inovação/renovação } \\
\text { Clientes que engrandecem a imagem da empresa } \\
\text { Vendas a novos Clientes }\end{array}$ & $\begin{array}{c}\text { 1.1.3.5 } \begin{array}{c}\text { Indicadores de } \\
\text { Inovação/renovação }\end{array} \\
\text { Proporção de novos produtos/serviços } \\
\text { Novos processos implementados }\end{array}$ & $\begin{array}{l}\text { Indicadores de Inovação/renovação } \\
\text { Gastos com treino e Formação } \\
\underline{\text { Diversidade }}\end{array}$ \\
\hline $\begin{array}{c}\text { 1.1.3.7 Indiccadores de Eficiência } \\
\text { Rentabilidade por cliente } \\
\text { Vendas por Cliente } \\
\text { Índice de Ganhos/perdas }\end{array}$ & $\begin{array}{l}\text { 1.1.3.8 Indiccadores de Eficiência } \\
\text { Proporção de pessoal de apoio. } \\
\text { Indice de Valores/atitudes }\end{array}$ & $\begin{array}{c}\text { 1.1.3.9 } \begin{array}{c}\text { Indiccadores de Eficiência } \\
\text { Efeito de Alavancagem }\end{array} \\
\frac{\text { Valor acrescentado/Empregado }}{\underline{\text { Lucro/Empregado }}}\end{array}$ \\
\hline $\begin{array}{l}\text { 1.1.3.10 Indicadores de Risco/Estabilidade } \\
\text { Proporção de Grandes Clientes. } \\
\text { Rácio de Cliente leais à empresa } \\
\text { Frequência de Ordens de Compra/Cliente }\end{array}$ & $\begin{array}{c}\text { 1.1.3.11 } \begin{array}{c}\text { Indicadores de } \\
\text { Risco/Estabilidade }\end{array} \\
\text { Idade da Organização } \\
\text { Rácio de Rotação do Pessoal de Apoio } \\
\text { Antiguidade da Organização }\end{array}$ & $\begin{array}{l}\text { Indicadores de Risco/Estabilidade } \\
\text { Rotação de Pessoal } \\
\text { Média de Idade do Pessoal } \\
\text { Antiguidade do Pessoal }\end{array}$ \\
\hline
\end{tabular}

Fonte: adaptado de http://www.sveiby.com/articles/MeasureIntangibleAssets.html , de Sveiby (1997), e de Gonçalves (2003).

No modelo $I A M$, desde o seu aparecimento até à atualidade não foram por nós identificadas praticamente quaisquer alterações - são conhecidas algumas aplicações, nomeadamente em meados da década de noventa do século passado, altura em que o mesmo foi referido com alguma frequência na literatura, mas parece-nos ter entrado em desuso a sua aplicação, a não ser em casos muito específicos. 


\section{2-O Modelo Balanced Scorecard (BSC)}

Um sistema de informação adequado pressupõe que os utilizadores na organização saibam qual a informação da qual necessitam, que a obtenham de forma regular e que a integrem na sua tomada de decisão. Neste sentido o BSC poderá ser encarado como um sistema que descreve a estratégia de forma clara, possibilita o equilíbrio e a integração entre os objetivos de curto e longo prazo, entre os resultados e os seus indutores, assim como entre medições objetivas e subjetivas, focalizando o desempenho organizacional sob quatro diferentes perspetivas: a Financeira; a do Cliente; a dos Processos Internos e a de Aprendizagem e Crescimento.

Estas perspetivas respondem a quatro questões centrais no modelo:

- para uma organização ser financeiramente bem sucedida, como deverá ser vista pelos seus acionistas?

- para a mesma atingir a sua missão, como deverá ser vista pelos seus clientes?

- para satisfazer os seus acionistas e clientes, quais os processos em que deverá atingir a excelencia?

- para alcançar a visão, como deverá a organização sustentar a sua capacidade de mudar e melhorar?

Segundo o conceito inicial (Kaplan e Norton, 1992), o BSC coloca a visão -"para onde iremos"- e a estratégia -"como iremos"- no centro do processo de gestão, tal como se mostra na Figura 3, estabelecendo objetivos e assumindo que as pessoas deverão adotar determinados comportamentos e ações que se tornam necessárias para os atingir.

Figura 3 - Modelo BSC.

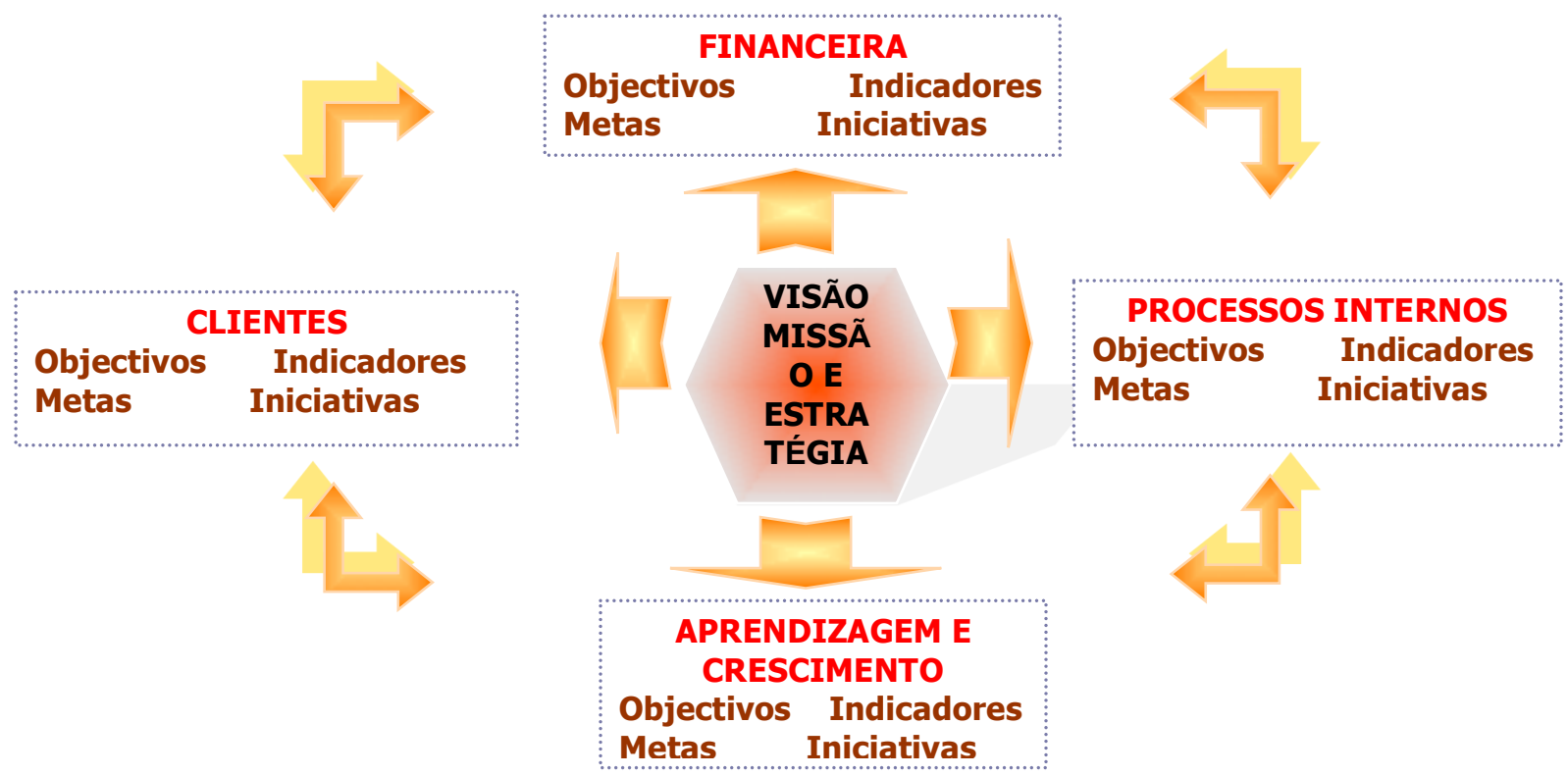

Fonte: adaptado de Kaplan e Norton (1997) e de http://www.keyassociados.com.br site consultado em Janeiro de 2006.

O BSC global de uma organização visa resumir a estratégia da mesma numa única página, através do mapa estratégico (Kaplan e Norton, 2004b), garantindo a simplificação do complexo documento estratégico que as empresas normalmente criam para explicar as linhas orientadoras da sua atividade. 
Outra finalidade do BSC relaciona-se com o facto de a comunicação da estratégia ser extensiva a toda a organização: o mapa estratégico dá-a a conhecer a todos os colaboradores, de forma a poderem contribuir para a concretização dos objectivos e a saberem como devem actuar. A não identificação dos colaboradores com os objetivos estratégicos será o primeiro sinal de desalinhamento e quebra da performance estratégica. Estas finalidades são ilustradas na Figura 4 - Mapa Estratégico, objetivos, indicadores, metas e iniciativas.

Figura 4 - Mapa Estratégico, objetivos, indicadores, metas e iniciativas.

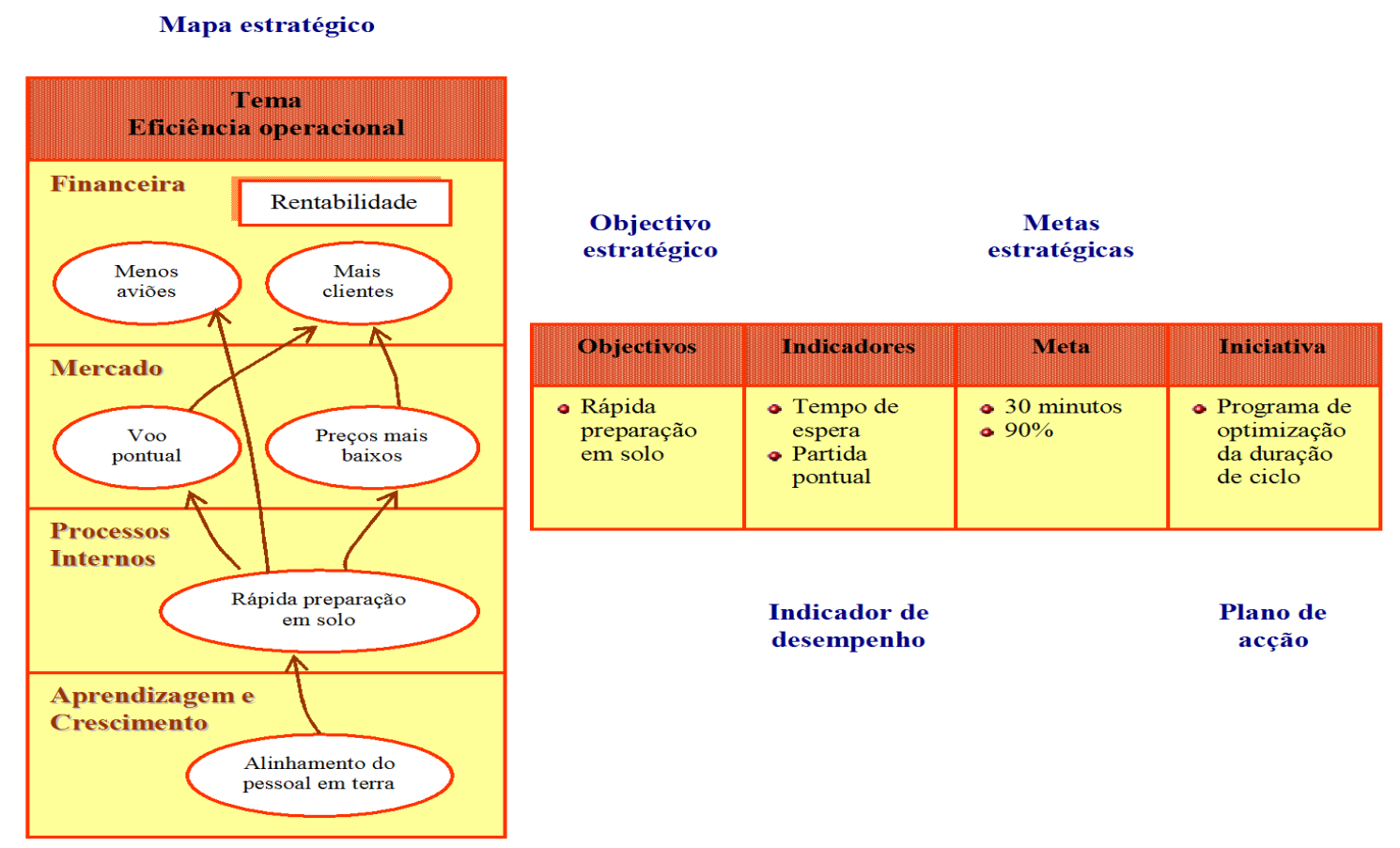

Fonte: http://www.bscol.com/invoke.cfm , site consultado em Janeiro de 2006.

Por último, o BSC pressupõe um acompanhamento periódico à execução da estratégia, permitindo no curto prazo que a organização possa comparar a sua performance com as metas definidas.

Toda e qualquer medida do BSC deve estar integrada numa cadeia de relações de causa e efeito que comunica a estratégia da empresa às unidades de negócios, devendo esta cadeia percorrer as perspetivas do BSC - estas vieram a revelar-se adequadas em diversas empresas e organizações, no entanto, não existe um número total de perspetivas a utilizar, ou seja, as empresas têm de encontrar as adequadas ao seu caso específico para alcançarem os seus objetivos, dependendo das circunstâncias do sector e da estratégia da unidade de negócios, sendo ainda possível agregar ou desagregar uma ou mais perspetivas complementares.

Tal como já foi referido o conceito sofreu uma evolução ao longo do tempo: - começou por ser aplicado numa ótica de avaliação do desempenho das organizações;

- posteriormente veio a ser encarado como um sistema de Gestão e de implementação e comunicação da estratégia; 
- de seguida foi atribuída uma grande ênfase à interligação entre o BSC e a gestão estratégica, nomeadamente ao nível da aprendizagem estratégica, a qual se relaciona com processos de inovação que inevitavelmente se prendem com o factor humano dentro das organizações;

- mais recentemente este instrumento tem vindo a ser aplicado numa perspectiva mais abrangente, tendo ultrapassado os limites internos de análise da organização, para uma perspectiva de análise e monitorização do sistema de gestão das organizações e da suas relações estratégicas com organizações externas.

O papel fundamental que o conhecimento vem revelando nas empresas, num contexto em que os trabalhadores são colaboradores do conhecimento e em que as estratégias devem ser executadas a todos os níveis na organização começou a ser claramente percebido e trabalhado pelas organizações (Quesado et al., 2014).

Desde o início do seu aparecimento até à atualidade os temas estratégicos do BSC descrevem a combinação de elementos intangíveis com os processos internos para se obterem resultados tanto intangíveis, como a fidelidade dos clientes, como tangíveis, tais como o crescimento de receita e da rendibilidade.

Desta forma, o BSC fornece como referência a medição de estratégias baseadas no conhecimento. Este facto relaciona-se com a perspectiva da aprendizagem e crescimento, a qual se focaliza na aquisição e desenvolvimento de conhecimentos, na construção da memória organizacional e na existência de um ambiente que permita o desenvolvimento das competências necessárias à evolução desejada pelas organizações.

Parece inquestionável que a finalidade de desenvolvimento das competências e de um ambiente organizacional que permita esse mesmo desenvolvimento, tem vindo a ser sucessivamente alargada dentro da organização - aparentemente iniciou-se com uma visão operacional da aplicação das potencialidades da ferramenta e foi-se sucessivamente, ao longo do tempo, alargando a visão da sua aplicação, funcionamento e potencialidades.

\section{3 - Modelo Skandia Navigator}

O Skandia Navigator, começou a ser desenvolvido em 1993 na empresa Skandia, mais concretamente na Skandia AFS, uma subsidiária desse grupo segurador. Esta organização tinha assegurado a colaboração de Edvinsson a partir de 1991, com o propósito de estabelecer um meio de medição dos processos geradores de valor.

Os estudos conduzidos na empresa deram origem àquilo que foi identificado como Capital Intelectual, e culminaram, em 1995, na realização de um relatório baseado nessa noção. 0 Navigator ou Balanced Scorecard do Capital Intelectual foi formalizado pelo autor (Edvinsson, 1997), e tem por base a estratégia da organização, tal como os modelos anteriormente referenciados, apresentando um modelo assente em quatro pilares, semelhantes às perspectivas do BSC, acrescentando-lhe no entanto um novo pilar: a perspetiva humana, a qual é considerada essencial na avaliação do desempenho organizacional e na possibilidade de gerar valor a longo prazo.

A representação clássica do modelo é apresentada na Figura 5, com as respectivas perspetivas, sua interligação com o factor tempo e os aspectos principais tangentes à noção de capital intelectual e respectiva gestão. 
Figura 5 - Modelo Skandia Navigator.

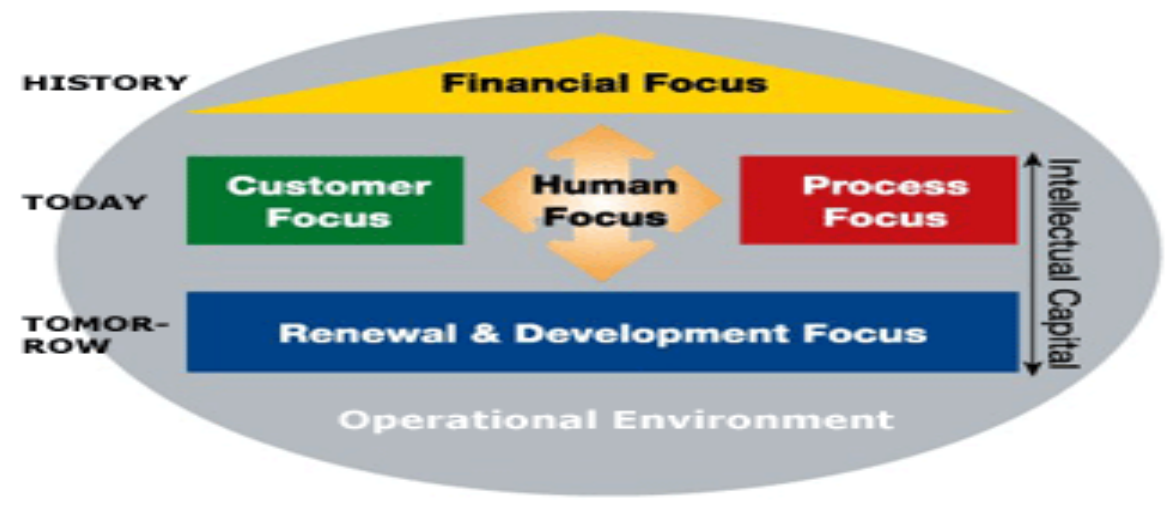

Fonte:http://www.valuebasedmanagement.net/methods_skandianavigator.html , consultado em Junho de 2011.

Nesta figura o foco financeiro, ou perspetiva financeira, está relacionado com os aspetos mais tradicionais, onde se incluem os dados históricos do desempenho. No presente, são considerados as relações com os clientes e os processos de produção e/ou prestação de um serviço, assim como os processos tecnológicos. A perspetiva focada no futuro é a de renovação e desenvolvimento, a qual se prende com a formação de recursos humanos, a inovação e temas relacionados com opções estratégicas da organização. Por fim, o foco nos recursos humanos assume um papel fundamental, ao influenciar, através da sua atuação, todas as outras perspetivas.

O modelo tem por base a definição de um conjunto de indicadores a partir dos factores críticos de sucesso e tendo como elemento central a estratégia prosseguida pela organização, que medem o seu desempenho e assumem o desígnio de aumentar o Capital Intelectual.

Segundo Russo (2009), este modelo de tipo Scorecard, teve difusão essencialmente nos países nórdicos. Parece-nos também que, apesar de ter tido no final dos anos noventa do século $X X$, vasta divulgação e até uma aplicação considerável, tem vindo a diminuir o seu impacto e a sua utilização, tal como acontece com o modelo analisado no ponto um.

Também não é conhecida uma evolução deste conceito no tempo, semelhante à que aconteceu com o BSC.

\section{4 - Análise da evolução dos modelos utilizados}

De acordo com o anteriormente referido, não muito distantes no tempo relativamente ao aparecimento do conceito de BSC, surgiram também as metodologias Intangible Assets Monitor (IAM), (Sveiby, 1987) e Skandia Navigator, (Edvinsson, 1997) cujos tipos de análise foram já resumidamente apresentadas. Estes modelos, embora diferentes, possuem algumas semelhanças entre si, cruzando-se os seus conceitos em diversos aspetos, nomeadamente quanto à relevância do que passou a designar-se depois da aparição dos mesmos por Capital Intelectual.

Os dois modelos identificados e aqui comparados com o BSC, têm ambos origem na Europa, mais concretamente na Suécia, tendo tido também uma considerável aplicação prática nomeadamente em meados da década de noventa do século $X X$, altura em que ambos os sistemas foram utilizados para reportar o valor do Capital Humano ou Capital Intelectual. 
No entanto essa utilização foi praticamente confinada à Suécia e países geograficamente próximos. Esta situação teve, mesmo assim, algumas exceções no referente ao modelo Skandia Navygator, o qual conseguiu uma divulgação e impacto mais alargado em termos geográficos: nomeadamente nos Estados Unidos e no Canadá através da utilização pelo Canadian Imperial Bank, numa fase inicial e posteriormente com a sua divulgação e alargamento a outras instituições.

Em 1993 o Conselho Sueco para Empresas de Serviços efectuou uma recomendação aos seus membros no sentido de aconselhar a utilização por parte das mesmas, de um número de indicadores que descrevessem o seu capital humano, nos seus relatórios anuais. A referida recomendação foi um dos contributos relevantes para as acções internacionais de recomendação para reporte do capital humano (ver Figura 6).

Figura 6 - Evolução dos Sistemas de Informação não Financeira de Gestão.

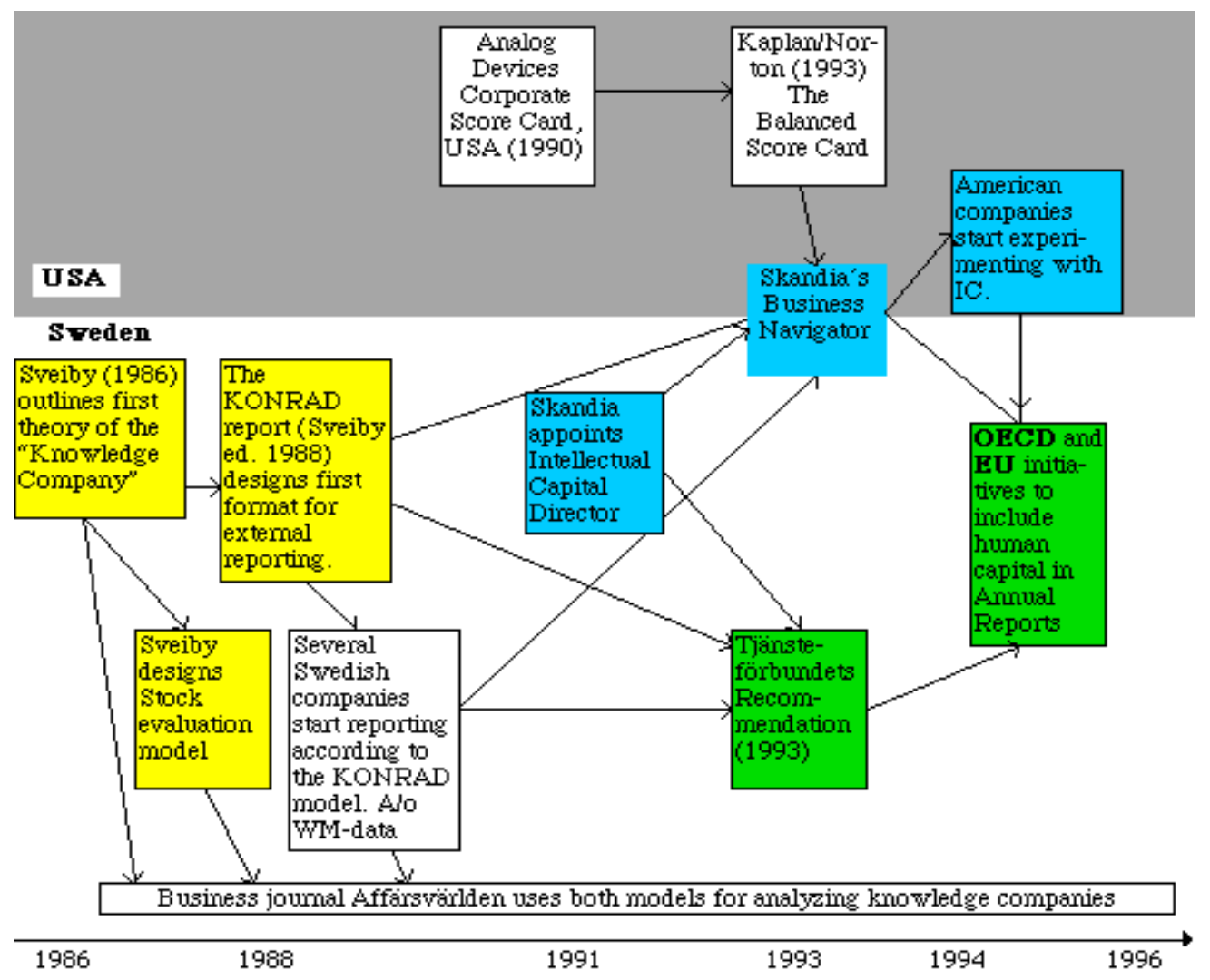

Fonte: Sveiby, Nilsson \& Strand, 1996 cit. in http://www.sveiby.com/articles/SwedishCoP.htm\#HRCA

As teorias do Capital Intelectual, tinham surgido no início dos anos noventa, na Skandia, e deram origem à subdivisão em Capital Humano e em Capital Estrutural (Gonçalves, 2003).

O Capital Humano corresponderia ao conjunto dos conhecimentos, competências e capacidade de inovação dos funcionários de uma organização - respeitando àquela parcela de elementos que no fim do dia sai da empresa e volta para casa - e que consequentemente, apesar de estar à disposição da organização, não é da sua propriedade.

Por outro lado, o Capital Estrutural incluiria bases de dados, softwares, estrutura organizacional, patentes, marcas, etc., que têm efeito na produtividade da organização, sendo estes elementos da sua efetiva propriedade. 
Os modelos apresentados neste trabalho, foram considerados como os mais significativos na medição e análise do capital intelectual das organizações (Russo, 2009), e diversos autores entendem que os modelos se influenciaram entre si: um exemplo disto é facto do próprio autor do Skandya Navigator assumir este modelo como um meio de encontrar uma maneira de medir a capacidade de criar valor, desenvolvendo assim a teoria do Capital Intelectual, que incorpora elementos quer do conceito de Konrad, quer do BSC.

O conceito de Konrad prende-se com a definição e utilização por parte de um grupo de gestores de organizações suecas de conceitos relacionados com o reporte dos ativos intangíveis. Este grupo iniciou a utilização de indicadores não financeiros com a finalidade de avaliar, monitorizar e divulgar os seus activos intangíveis, tendo sido um dos elementos percursores das teorias do Capital Intelectual. O objetivo deste grupo de gestores era o de encorajar as organizações do conhecimento a melhorar os seus relatos para o exterior e a efectuar uma avaliação mais ajustada do valor real destas organizações.

Considerando a análise cronológica efetuada neste trabalho, a partir do final da década de noventa do século XX, não foram identificadas quaisquer evoluções dos conceitos iniciais dos dois modelos aqui comparados com o BSC.

Pelo contrário, em relação ao BSC, em finais do século XX e inícios do XXI, autores como Kanji (2003), e Kanji e Sá (2001, 2002), afirmam que o mesmo é na altura utilizado de duas formas distintas pelas organizações: como um sistema de informação que suporta a focalização dos responsáveis e também como um sistema de gestão estratégica baseado nos critérios apresentados por Kaplan e Norton.

Neste ponto parece-nos pertinente analisar como e porquê uma inovação se difunde de umas organizações a outras. Não será alheio a este fenómeno o factor moda nas atividades de Gestão (desde o Controlo de Gestão - atividade essencialmente relacionada com as práticas contabilísticas de Gestão, até à definição e monitorização da estratégia). No entanto, mesmo este factor moda ou tendência é justificado por ser maioritariamente utilizado por alguma razão: ou por razões de legitimação interna por parte da gestão da organização, ou de legitimação do seu sistema de gestão perante os interessados na evolução da mesma, ou mesmo por mimetismo com outras organizações (Abrahamson e Fairchild (1999), Malmi (2001), Ax e Bjornenak (2005)).

Parece-nos que ambos os argumentos invocados - legitimação ou mimetismo - terão de estar relacionados com os resultados obtidos em diversas organizações (Kaplan e Norton, 2000a), aliás amplamente comunicados quer pelos autores, quer por empresas de consultadoria, quer por empresas de tecnologias de informação. O efeito moda pode estar aqui relacionado com o facto de em diversas organizações o modelo ter obtido algum grau de êxito - o que atraiu outros utilizadores, mas também com a ampla comunicação desse êxito efectuada por diversos meios, tais como (Saraiva, 2011a; Saraiva e Alves, 2015):

- iniciativas levadas a cabo pelos autores da metodologia, relacionadas com atribuição de prémios e incentivos a organizações que a implementaram e que obtiveram êxito;

- existência de numerosas consultoras na área da implementação e desenvolvimento do BSC, algumas avalizadas pelos autores da metodologia;

- divulgação de soluções informáticas de aplicação do BSC;

- a realização de Seminários, cursos de formação, workshops, conferências e jornadas sobre o tema; 
- os papers apresentados em congressos das áreas de Gestão e de Contabilidade de Gestão e até da área de Gestão de Recursos Humanos;

- os programas de formação apresentados pelas instituições de ensino (mormente de ensino superior e entidades de formação para empresários);

- o facto de qualquer obra actual de âmbito escolar das áreas de Gestão, Gestão de Recursos Humanos e Contabilidade de Gestão, dedicarem uma parte da sua estrutura à apresentação e ao funcionamento do BSC.

Todos estes factores parecem contribuir para o que pode entender-se como um processo de difusão de inovação (Martínez e Herédia, 2003), e consequentemente de alteração das práticas de gestão e de contabilidade de gestão. Um dos principais argumentos para esta mudança foi a necessidade de medidas de gestão e de contabilidade de gestão de carácter não financeiro. Neste campo, um dos conceitos mais importantes é sem qualquer margem para dúvida o BSC, (Kaplan e Norton, 1992; Butler et al., 1997; Silk, 1998).

No que concerne à mudança na contabilidade de gestão e suas práticas, as medidas não financeiras assumem um papel de relevo (Bhimani, 1993; Lapsley and Mitchell, 1994). Os processos de medição passaram a poder incluir mais do que os aspectos meramente tangíveis uma nova-ordem surgiu a partir da utilização de medidas não financeiras.

Por outro lado, haverá um diferente aspecto a considerar: a mudança que se estabelece dentro das organizações que implementam o BSC.

Pettigrew e Whipp (1991), definem um quadro teórico com a finalidade de entender a mudança estratégica, encarando-a como um processo contínuo. Os autores afirmam que existem três diferentes dimensões inter-relacionadas a considerar para se perceber a mudança estratégica: o conteúdo da estratégia, o processo de mudança e o contexto de actuação em a mudança acontece. As três dimensões relacionam-se na medida em que o contexto tem de ser considerado para entender o conteúdo e os processos de mudança, e o modelo pode ser usado para estudar mudanças que não apenas as estratégicas. Este modelo pode também servir para aferir se $O$ BSC se enquadra como uma ferramenta indutora de mudança dentro das organizações. Assim poderá ser apontada a fase de desenho do BSC, como a dimensão de conteúdo da mudança, a dimensão de processo corresponderá à implementação do BSC, em que o desenho do mesmo é integrado e adaptado à organização; a dimensão de contexto corresponderá à utilização e funcionamento do BSC. Esta última dimensão prende-se com a forma como o desenho do BSC é aplicado na prática, pois a sua utilização dependerá muito da forma como foi implementado (Papalexandris et al., 2004, 2005). O factor tempo também é importante - a mudança leva tempo a operar-se e mesmo que o desenho e a implementação do BSC demore apenas alguns meses, é possível que passem vários anos até a totalidade do processo estar consolidado em toda a organização - este ponto prende-se com a continuidade do processo de mudança estratégica (Venkatraman e Gering, 2000 e Sioncke, 2005).

Anthony e Govindarajan (2001), afirmam que o BSC se tornou um sistema de avaliação da performance que tem por finalidade a implementação de estratégias. O conceito inicialmente introduzido por Kaplan e Norton tem finalidades no âmbito do controlo de gestão, posteriormente a definição alargou-se ao incluir tanto a natureza do produto final como a actividade de controlo de gestão. 
O que se verificou na prática foi que os responsáveis das organizações que adoptavam o BSC, utilizavam o conceito para realinhar as suas unidades de negócio, unidades de serviço partilhado, equipas e indivíduos em torno de metas e objectivos gerais (Kaplan e Norton, 2004a).

A partir da base de trabalho estabelecida por Kaplan e Norton, académicos e responsáveis por organizações em todo mundo, começaram a interessar-se pelo conceito e a estudá-lo, contribuindo para o seu aperfeiçoamento. Na Europa, América Latina e Austrália surgiram empresas de consultoria certificadas pelo Balanced Scorecard Collaborative.

Durante os anos seguintes Kaplan e Norton acompanharam diversas organizações que implementaram o BSC, constatando que as mesmas conseguiam resultados importantes em períodos relativamente curtos e que os responsáveis pelas organizações suportavam as suas estratégias no conceito de relação de causa e efeito representado nos mapas estratégicos que implementavam nas mesmas (Kaplan e Norton, 2000a, 2004a, 2004b).

A difusão do modelo junto quer dos meios académicos - onde apesar de tudo, também surgem visões críticas - Norreklit $(2000,2003)$, quer dos meios profissionais, parece dever-se, em parte, à forma como é promovido e suportado pelos autores de referência: Kaplan e Norton não só publicam vasta literatura sobre o tema como também promovem a criação de empresas de assessoria que por sua vez muito contribuem para a sua disseminação; com a criação do Balanced Scorecard Collaborative, Inc., surge toda uma plêiade de serviços, conferências, formação, certificação, standards de implementação, softwares, em torno da aplicação e utilização do BSC. Surge também a iniciativa promovida pelos autores, BSCOL "Hall of Fame", que confere um prémio a organizações que tenham obtido bons resultados com a implementação e utilização do conceito.

Releva-se ainda a questão da ligaçação desta ferramenta aos sistemas de gestão da qualidade e à introdução de inovações nas organizações, associada à implementação destes sitemas (Saraiva e Gabriel, 2016).

A referida disseminação no mundo profissional tem sido sem dúvida essencial para a evolução desta ferramenta - vejam-se as evoluções apresentadas em 2008 por Kaplan e Norton, ao assumir o BSC como um sistema que se coloca já num plano em que se gere a estratégia e as relações com o exterior (Kaplan e Norton, 2008), e em 2010, por Kaplan, Norton e Rugelsjoen, em que o BSC surge como um sistema de gestão de alianças estratégicas, por parte das organizações. Mais uma vez, esta última aplicação do BSC surge a partir da aplicação numa organização da área farmacêutica, para estabelecer uma parceria, surgindo a noção do "Scorecard de Parceria ou de Aliança" (Kaplan, Norton e Rugelsjoen, 2010). No artigo de 2010 são também referidas outras experiências neste tipo de utilização, por outras empresas em diferentes países.

Este último campo de actuação do BSC surge claramente como um resultado da aplicação efectuada na prática - a qual vem redefinir o conceito - alargando-o aos aspectos relacionados com a gestão estratégica das relações externas entre organizações.

Assim a análise da utilização e da evolução deste instrumento de gestão parece assumir relevância a vários níveis, isto porque parece evidente que ao longo do tempo a utilização dada ao BSC fez evoluir o mesmo e o seu conceito, e vice-versa, a evolução do BSC também fez alterar o campo de aplicação do mesmo, a avaliar pelas evoluções mais recentes. Além disso parece também pertinente a ideia, face ao apresentado, que a utilização generalizada deste instrumento pelos gestores na atualidade, influenciou a mudança nas práticas de Gestão, ao surgir 
actualmente como um elemento tão regular na definição de estratégias nas organizações e ao nível do conhecimentos, em geral, como as clássicas análise SWOT ou a matriz BCG (Saraiva, 2011a).

\section{Conclusões}

Pelo argumentado anteriormente poder-se-á concluir que a evolução histórica do BSC, se relaciona com a evolução das aplicações em que a ferramenta tem sido utilizada: a sua abrangência e profundidade foi-se alargando através do potencial de utilização verificado na prática.

Por outro lado, as práticas de Gestão e de Contabilidade de Gestão, são atualmente profundamente marcadas quer pela utilização de medidas não financeiras, quer pelo recurso à utilização de relações de causa e efeito. Tanto umas como outras são amplamente consideradas pelos modelos do BSC, do IAM e do Skandia Navigator. No entanto, atualmente continua a utilizar-se e a referir-se, de uma forma praticamente global, o primeiro, enquanto que os últimos, também de maneira geral, parecem ter sido utilizados durante um período de tempo relativamente limitado e com muito menor intensidade que o primeiro.

Parece-nos ainda que a intensidade de utilização do BSC, tal como foi documentada ao longo deste trabalho, poderá ter sido determinante na prevalência do BSC em detrimento de outros modelos.

Poderá retomar-se neste ponto a ideia já referida e apresentada (Kaplan e Norton, 1992; 2004a; 2004b), de a informação financeira ser o culminar ou o resultado de acções e tarefas desenvolvidas nas e pelas organizações, tendo a contabilidade sido encarada como o sistema de informação mais tradicionalmente desenvolvido pelas organizações (por razões de ordem legal, fiscal ou de avaliação do funcionamento organizado das mesmas), e tornando-se a base de avaliação tradicional dos resultados. No entanto verifica-se que nem sempre estes indicadores têm aderência à estratégia.

Assim, depois de identificado este tipo de informação (estratégica), a organização deve preocupar-se com a sua utilização: o que passa não só por definir uma estratégia, mas também por implementá-la.

Verificou-se que a referida evolução surgiu como consequência das aplicações do BSC efetuadas no mundo empresarial. Também nas suas evoluções mais recentes, a aplicação do conceito base de BSC a uma situação diferenciada e mais voltada para o exterior da organização do que até aí se tinha realizado, veio permitir todo um novo campo de actuação ao BSC, originando um conceito diferenciado do que até aí existia.

Comparou-se ainda a evolução sofrida pelo BSC com o facto das outras metodologias analisadas, contemporâneas ao seu aparecimento e com o mesmo tipo de características, aparentemente não terem passado pelo mesmo género de evolução - o que, cruzado com o facto de as outras metodologias não terem tido, ao longo do tempo, uma aplicação tão intensiva como a do BSC, parece indicar que o factor utilização pratica foi extremamente importante e determinante para a evolução do conceito.

Por fim tentou estabelecer-se que a metodologia, por ser amplamente utilizada, conduziu a alterações importantes nas práticas de Gestão e de Contabilidade de gestão, ao constituir-se como um dos principais instrumentos de mudança no que respeita à utilização de medidas não 
financeiras como indicadores de Gestão de elevada importância e relevância. Também no que respeita à mudança interna nas organizações, como se verificou, é um facilitador e promotor da mesma.

\section{BIBLIOGRAFIA}

ABRAHAMSON, E. e FAIRCHILD, G. (1999). "Management Fashion: Lifecycles, Triggers, and Colective Learning Processes", Administrative Science Quarterly, December, Vol. 44, n. 4, pp.708-740.

ANTHONY, R. e GOVINDARAJAN, V. (2001). Management control systems. Boston, McGraw-Hill.

AX, C. e BJORNENAK, T. (2005). "Bundling and Diffusion of Management Accounting Innovations - The Case of the Balanced Scorecard in Sweden", Management Accounting Research, March, Vol. 16, n.o 1, pp.1-20.

BHIMANI, A., (1993). "Indeterminacy and the specificity of accounting change: Renault 1898-1938", Accounting, Organizations and Society, 18(1), pp.1-39.

BUTLER, A., LETZA, S. e NEALE, B., (1997). “Linking the balanced scorecard to strategy", Long Range Planning, 30(2), pp.242-253.

CRUZ, C.P. (2006). Balanced Scorecard - Concentrar uma Organização no que é Essêncial, Vida Económica, Porto.

DOWNING, L.; (Novembro/Dezembro 2000). "Progress report on the Balanced Scorecard: a global users'survey", Balanced Scorecard Report, Harvard Business School Press, volume 2, n. 6;

GONÇALVES, D. (2003). “Capital Intelectual: considerações sobre a sua valorização”, in Jornal do Técnico de Contas e da empresa, no 452, pp.182-188.

KANJI, G. (2003), "Performance measurement system", Total Quality Management, Vol. 13, No. 5, August, pp. 715728.

KANJI, G. e SÁ, P. (2002). “Kanji's business scorecard”, Total Quality Management, Vol. 13, No. 1, January, pp.13-27.

KANJI, G. e SÁ, P. (2001). "Kanji's business scorecard", Total Quality Management, Vol. 12, No. 7, December, pp. 898905.

KAPLAN, R.; NORTON, D. (2010). "Managing Alliances with the Balanced Scorecard", Harvard Business Review, January-February, pp. 114-120.

KAPLAN, R.; NORTON, D. (2008). "Mastering the Management System", Harvard Business Review, January, Vol. 86, n.․ 1, pp. 62-77.

KAPLAN, R.; NORTON, D. (2007). "Using the Balanced Scorecard as a Strategic Management System", Harvard Business Review, July/August, Vol. 85, n. 7/8, pp.150-161.

KAPLAN, ROBERT S., NORTON, DAVID P., (2004a). Mapas estratégicos convertendo activos intangíveis em resultados tangíveis, Campus, Rio de Janeiro;

KAPLAN, Robert S.; NORTON, David P. (2004b). "How Strategic Maps Frame an Organization's Objectives", Financial Executive, March/April, Vol. 20, n. 2, pp. 40-45.

KAPLAN, R. S. e NORTON, D. P., (1997). A estratégia em acção: Balanced Scorecard, Campus, Rio de Janeiro.

KAPLAN, R. e NORTON, D. (1992). "The Balanced Scorecard - Measures that Drive Performance", Harvard Business Review, Jan-Feb, pp.71-79. 
Karl-Erik Sveiby. Paper for PEI Conference in Stockholm 25 October 1996. Updated April 2001.

LAPSLEY, I. and MITCHELL, F., (1994). "Management accounting research: the change agenda", Management Accounting Research, 5, pp.215-219.

MALMI, T. (2001). "Balanced Scorecards in Finnish Companies: A Research Note", Management Accounting Research, June, Vol. 12, n. 2, pp.207-220.

MARTÍNEZ RAMOS, M.; HEREDIA ÁLVARO, J. (2003). “El Balanced Scorecard. Estudio del Proceso de Implantación en una Mediana Empresa", Revista Iberoamericana de Contabilidad de Gestión, Julio/Diciembre, Vol. I, n.o 2, pp.147168.

NORREKLIT, H. (2003). "The balanced scorecard: what is the score? A rhetorical analysis of the balanced scorecard", Accounting, Organizations and Society, Vol. 28, No. 6, August, pp. 591-619.

NORREKLIT, H. (2000). "The balance on the balanced scorecard - a critical analysis of some of its assumptions", Management Accounting Research, Vol.11, No.1, March, pp. 65-88;

PAPALEXANDRIS, A., IOANNOU, G., PRASTACOS, G. e SODERQUIST, K. (2005). “An integrated methodology for putting the balanced scorecard into action", European Management Journal, Vol. 23, No. 2, April, pp.214-227.

PAPALEXANDRIS, A., IOANNOU, G. e PRASTACOS, G. (2004). "Implementing the balanced scorecard in Greece: a software firm's experience", Long Range Planning, Vol. 37, No. 4, August, pp.351-366.

PETTIGREW, A. e WHIPP, R. (1991). Managing change for competitive success, Cambridge, Blackwell.

QUESADO, P.R., RODRIGUES, L.L. e AIBAR GUZMÁN (2014). “EL CUADRO DE MANDO INTEGRAL, EL ACTIVITY BASED COSTING Y LA ACTIVITY BASED MANAGEMENT: UN ESTUDIO EMPÍRICO DE SU INTEGRACIÓN", RIC - Revista de Informação Contábil, Vol. 8, no 1, Jan-Mar, pp. 71-94. ISSN 1982-3967.

QUESADO, Patrícia Rodrigues (2010). Factores Determinantes de la Implementación del Cuadro De Mando Integral en Organizaciones Públicas y Privadas Portuguesas, Tese de Doutoramento, Universidad de Santiago de Compostela, Facultad de Ciencias Económicas y Empresariales, Departamento de Economía Financiera y Contabilidad, Santiago de Compostela.

QUESADO, Patrícia Rodrigues (2005). O Contributo do Balanced Scorecard para a Gestão Estratégica de Custos: uma Análise Empírica às Grande Empresas Portuguesas, Dissertação de Mestrado, Universidade do Minho, Braga.

RUSSO, J. (2009). Balanced Scorecard para PME, Lídel - edições técnicas, Lda. Lisboa-Porto.

SANTOS, R.A. (2006). Balanced Scorecard em Portugal, Visão, Estratégia e Entusiasmo. Gestão plus Edições, Cascais.

SARAIVA, H. e GABRIEL, V. (2016). "Determinantes da Certificação da Qualidade nas Autarquias Portuguesas", Revista Portuguesa de Estudos Regionais, no 41, pp.35-48.

SARAIVA, H.I.B. e ALVES, M.C.G. (2015). "The use of the Balanced Scorecard in Portugal: Evolution and effects on management changes in Portuguese large companies", Tékhne, Volume 13, Issue 2, July-December 2015, pp. 82-94. doi:10.1016/j.tekhne.2016.04.001

SARAIVA, H. (2011a). "The Balanced Scorecard: The Evolution of the Concept and Its Effects on Change in Organizational Management", EBS Review, 28, pp.53-66. ISSN 1406-0264.

SARAIVA, H. (2011b). "A evolução do Balanced Scorecard - efeito da sua aplicação prática", Revista Portuguesa de Contabilidade, V.I, no2, pp. 265-276.

SILK, S. (1998)." Automating the balanced scorecard", Management Accounting (US), pp.38-44. 
SIONCKE, G. (2005), "Implementation of a balanced scorecard in a care home for the elderly: Useful or not?", Total Quality Management, Vol. 16, No. 8/9, pp.1023-1029.

SVEIBY, KARL ERIK (1997). "The Intangible Assets Monitor", Journal of Human Resource Costing \& Accounting, Vol. 2 Iss: 1, pp. $73-97$.

VENKATRAMAN, G. e GERING, M. (2000). "The balanced scorecard", Ivey Business Journal, Vol. 64, No. 3, January/February, pp.10-14.

VIEDMA MARTÍ, J. (2002). "Un Nuevo Paradigma Emergente de Capital Intelectual”, XII Jornadas Luso-Espanholas de Gestão Científica, UBI, Covilhã.

\section{- Sites consultados:}

http://www.bscol.com/invoke.cfm, site consultado em Janeiro de 2006 e em Dezembro de 2015.

http://www.keyassociados.com.br, site consultado em Janeiro de 2006.

https://www.thepalladiumgroup.com, site consultado em Janeiro de 2011.

http://www.valuebasedmanagement.net/methods_skandianavigator.html, site consultado em Junho de 2016.

http://www.sveiby.com/articles/MeasurelntangibleAssets.html, site consultado em Julho de 2011.

http://www.sveiby.com/articles/companymonitor.html, site consultado em Julho de 2011.

http://www.emeraldinsight.com/journals.htm , site consultado em Julho de 2011. 